\title{
Critical Networks Exhibit Maximal Information Diversity in Structure-Dynamics Relationships
}

\author{
Matti Nykter ${ }^{1,2}$, Nathan D. Price ${ }^{2,4}$, Antti Larjo ${ }^{1}$, Tommi \\ Aho $^{1}$, Stuart A. Kauffman ${ }^{3}$, Olli Yli-Harja ${ }^{1}$, Ilya Shmulevich ${ }^{2, *}$ \\ ${ }^{1}$ Institute of Signal Processing, Tampere University of Technology, Tampere, Finland \\ ${ }^{2}$ Institute for Systems Biology, Seattle, WA, USA \\ ${ }^{3}$ Institute for Biocomplexity and Informatics, \\ University of Calgary, Calgary, AB, Canada \\ ${ }^{4}$ Department of Chemical and Biomolecular Engineering, \\ University of Illinois, Urbana, IL, USA \\ * To whom correspondence should be addressed (ishmulevich@systemsbiology.org)
}

(Dated: October 24, 2018)

\begin{abstract}
Network structure strongly constrains the range of dynamic behaviors available to a complex system. These system dynamics can be classified based on their response to perturbations over time into two distinct regimes, ordered or chaotic, separated by a critical phase transition. Numerous studies have shown that the most complex dynamics arise near the critical regime. Here we use an information theoretic approach to study structure-dynamics relationships within a unified framework and show that these relationships are most diverse in the critical regime.
\end{abstract}

PACS numbers: Valid PACS appear here 
The structural organization, or topology, of a complex system strongly constrains the range of dynamical behaviors available to the system. Understanding structure-dynamics relationships in networks is a major goal of complex systems research [1, 2]. However, general principles behind such relationships are still lacking, in part due to the lack of sufficiently general formalisms for studying structure and dynamics within a common framework. Numerous relationships between specific structural and dynamical features of networks have been investigated [3, 4, 5, 6, 6]. For example, structure can be studied by means of various graph-theoretic features of network topologies such as degree distributions [8] or modularity [9], or in terms of classes of updating rules that generate the dynamics [6, 7]. Aspects of dynamical behavior include transient and steady-state behavior and the response of the system to perturbations [3, 10].

Relating structure to dynamics is important for understanding emergent behaviors because the structure in a complex system directly affects emergent properties such as robustness, adaptability, decision-making and information processing [3, 11]. An important aspect of many complex dynamical systems is the existence of two dynamical regimes, ordered and chaotic, with a critical phase transition boundary between the two [12, 13, 14]. These regimes profoundly influence emergent dynamical behaviors, and can be observed in different ensembles of network structures. Networks operating in the ordered regime are intrinsically robust, but exhibit simple dynamics. This robustness is reflected in the dynamical stability of the network both under structural perturbations and transient perturbations. Contrary to this, networks in the chaotic regime are extremely sensitive to small perturbations, which rapidly propagate throughout the entire system and hence fail to exhibit a natural basis for robustness and homeostasis. The phase transition between the ordered and chaotic regimes represents a tradeoff between the need for stability and the need to have a wide range of dynamic behavior to respond to a variable environment. It has long been hypothesized that biological networks operate near this phase transition [10]. Recent evidence suggests that biological networks are not chaotic [15, 16].

A theme that has emerged in many contexts in systems theory is that complex systems operating near the phase transition exhibit maximally "interesting" dynamics [17, 18, 19, 20, 21, 22]. Complex coordination of information processing seems to be maximized near the phase transition. Information theory provides a common lens through which we can study both structure and dynamics of complex systems within a unified framework. 
Indeed, since network structures as well as their dynamic state trajectories are objects that can be represented on a computer, the information encoded in both can be compared and related. Unlike Shannon's information, which is defined in terms of distributions, Kolmogorov complexity is a suitable framework for capturing the information embedded in individual objects of finite length [23].

Recent developments in information theory have demonstrated that Kolmogorov complexity can be used to define an absolute information distance between two objects [24, 25], called herein universal information distance. This distance metric is universal in that it can be applied to any objects that can be stored on a computer (e.g. networks or genome sequences), and uniquely specifies an information distance without parameters of any kind. Thus, it is suitable for comparing the information content of two objects. Although this distance is, like the Kolmogorov complexity itself, uncomputable, it can be approximated by real-world data compressors (herein, gzip and bzip2) to yield the normalized compression distance (NCD) [25], defined as

$$
N C D(x, y)=\frac{C(x y)-\min \{C(x), C(y)\}}{\max \{C(x), C(y)\}},
$$

where $C(x)$ is the compressed size of $x$ and $x y$ is the concatenation of the strings $x$ and $y$. Cilibrasi and Vitányi [26] have demonstrated that the NCD can be used for clustering using a real-world compressor with remarkable success, approximating the provable optimality of the (theoretical) universal information distance.

Herein we apply the NCD to various classes of networks to study their structure-dynamics relationships. The proposed methods can be applied in principle to any model class that can be represented on a computer. We can represent the state of a network by a set of $N$ discrete-valued variables, $\sigma_{1}, \sigma_{2}, \ldots, \sigma_{N}$. Examples include Boolean networks $\left(\sigma_{n} \in\{0,1\}\right)$ [10, 12, 27], ternary networks $\left(\sigma_{n} \in\{0,1,2\}\right)$, and so forth, depending on the level of detail desired.

To each node $\sigma_{n}$ we assign a set of $k_{n}$ nodes, $\sigma_{n_{1}}, \sigma_{n_{2}}, \ldots, \sigma_{n_{k_{n}}}$, which control the value of $\sigma_{n}$ through the equation $\sigma_{n}(t+1)=f_{n}\left(\sigma_{n_{1}}(t), \sigma_{n_{2}}(t), \ldots, \sigma_{n_{k_{n}}}(t)\right)$. In the case of Boolean networks, we choose the functions $f_{n}$ randomly from the set of all possible Boolean functions such that for each configuration of its $k_{n}$ arguments, $f_{n}=1$ with probability $p$, known as the bias. The average of $k_{n}$, denoted by $K$, is called the average network connectivity. Here, we consider two types of wiring of nodes: 1 ) random, where each node has exactly $K$ inputs 
chosen randomly; and 2) regular, where nodes are arranged on a regular grid such that each node takes inputs from its $K$ neighbors. For random wiring the critical phase transition curve is defined by $2 K p(1-p)=1[13]$. Thus, for $p=0.5$ (unbiased) $K=1,2$, and 3 correspond to ordered, critical, and chaotic regimes, respectively.

Making use of the universal information distance as approximated by the NCD, we are able to compare networks without reducing them to arbitrary sets of features (e.g., graph properties). Indeed, the NCD uses the information and regularities embedded in the network structure to pick up the relative differences in the structural complexities of the networks. In order for NCD to most effectively capture structural differences, a network structure representation that is most amenable to compression (i.e. approximating the Kolmogorov complexity as well as possible) should be used. For example, for Boolean networks, we represent the connections by distances along an arbitrary linear arrangement of the nodes, making the regularities in the network structure more easily observable. See supplementary material for details on the encoding of network structures.

To illustrate this, we generated six Boolean network ensembles $(N=1000)$ with two different wiring topologies: random and regular, each with $K=1,2$, or 3. As Figure 1 illustrates, all of the different ensembles considered are clearly distinguishable.

To demonstrate that the NCD is able to capture meaningful structural relationships between networks, we applied it to compute the pairwise distances between the metabolic networks of 107 organisms from the KEGG database [28] (see supplementary material for details). The resulting phylogenetic tree, generated using the complete linkage method, is shown in Figure 2. The organisms are clearly grouped into the three domains of life. The archaea and eukaryotes both separate into distinct branches of phylogenetic tree based on the information content of their metabolic networks. The bacteria form three distinct branches, with parasitic bacteria encoding more limited metabolic networks separating from the rest, as has been observed previously [29]. The fact that the phylogenetic tree reproduces the known evolutionary relationships suggests that the NCD successfully extracts structural information embedded in networks.

We used NCD to study the relationship between structural information and dynamical behavior within a common framework. Within each of the above 6 ensembles, we generated 150 networks and calculated the NCD between all pairs of network structures and between their associated dynamic state trajectories. After running the network dynamics 100 steps 
from a random initial state to ensure that the network is not in a transient state, state trajectories were collected for 10 consecutive time steps and the states were concatenated into one vector (see supplementary material for details on the encoding of dynamics). As is the case with the structure of the networks, NCD is expected to detect the regularities in the state trajectories and thus uncover their intrinsic similarities. The relationship between structure and dynamics was visualized by plotting the structure-based NCD versus the dynamics-based NCD for pairs of networks within each ensemble (Figure 3). All network ensembles were clearly distinguishable based on their structural and dynamical information. It is also interesting to note that within-ensemble distances increase as dynamical complexity increases. Similarly, as the structure gets more complex, from regular to random wiring, or with increasing in-degree, the within-ensemble distances increase.

The critical ensemble ( $K=2$, random wiring) exhibited a distribution that is markedly more elongated along the dynamics axis as compared to the chaotic and ordered ensembles, supporting the view that critical systems exhibit maximimal diversity. The wide spread of points for the critical network ensemble in Figure 3 shows that their dynamics range between those of ordered and chaotic ensembles. Indeed, very different network structures can yield both relatively similar and dissimilar dynamics, thereby demonstrating the dynamic diversity exhibited in the critical regime. Thus, the universal information distance provides clear evidence that the most complex relationships between structure and dynamics occur in the critical regime.

In summary, we have demonstrated that NCD is a powerful tool for extracting structuredynamics relationships. It is fascinating that the analyses presented herein are possible using only the compressibility of a file encoding the network or its dynamics without needing to select any particular network parameters or features. This approach allows us to study, under a unified information theoretic framework, how a change in structural complexity affects the dynamical behavior, or vice versa.

\section{Acknowledgments}

This work was supported by NIH-GM070600 (IS, SAK), NIH-GM072855 (IS), NIH-P50 GM076547 (IS), Academy of Finland (project No. 213462 OY-H, No. 120411 and No. 122973 MN), American Cancer Society PF-06-062-01-MGO (NDP). The authors are grateful 


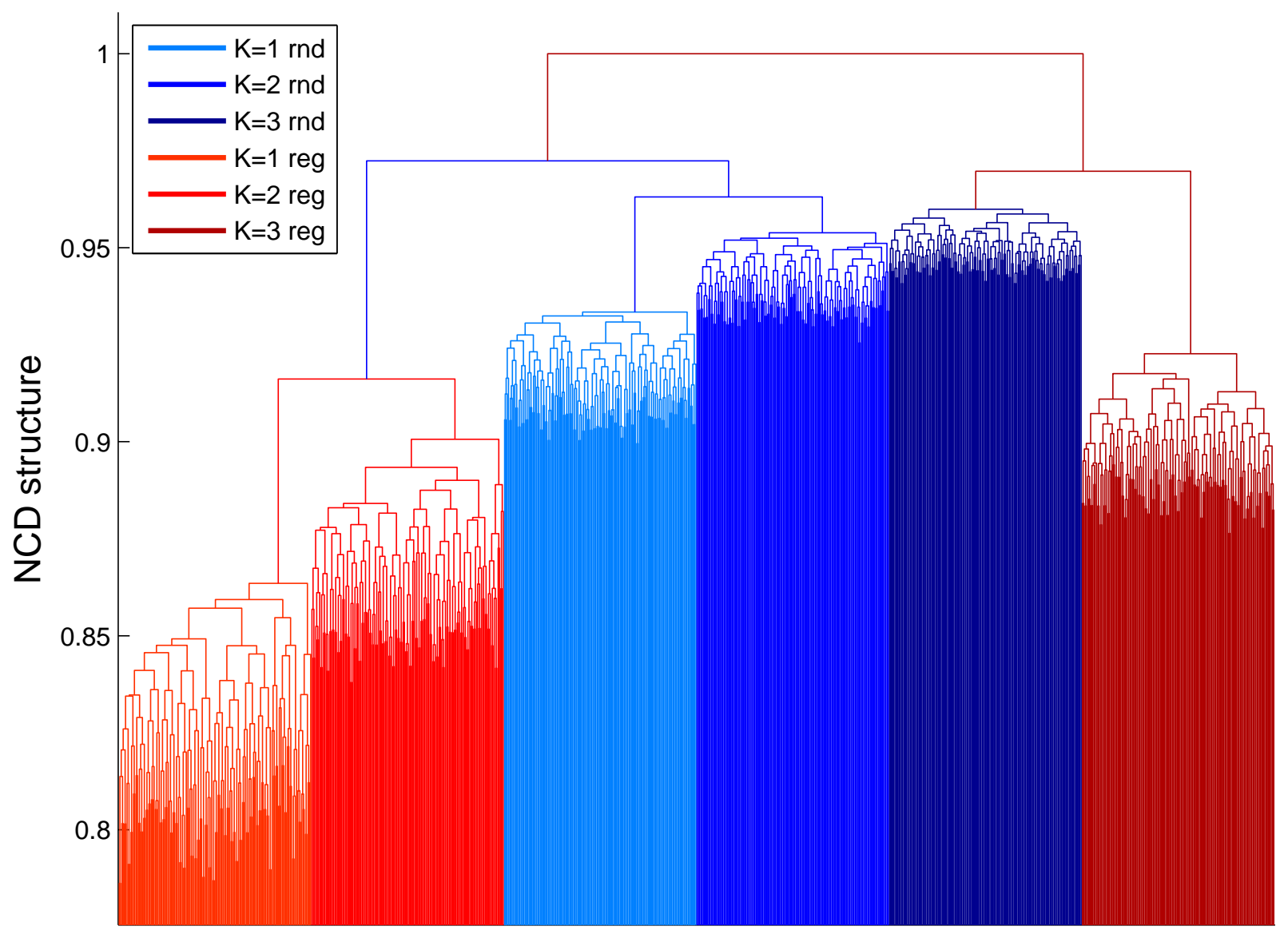

FIG. 1: Six ensembles of random Boolean networks $(K=1,2,3$ each with random or regular topology; $N=1000$ ) were used to generate 30 networks from each ensemble. The normalized compression distance (NCD) was applied to all pairs of networks. Hierarchical clustering with the complete linkage method was used to build the dendrogram from the NCD distance matrix (see supplementary material for details on clustering). Networks from different ensembles are clustered together, indicating that intra-ensemble distances are smaller than inter-ensemble distances. 
to Leroy Hood, David Galas, Jared Roach, and Alok Srivastava for useful discussions. 

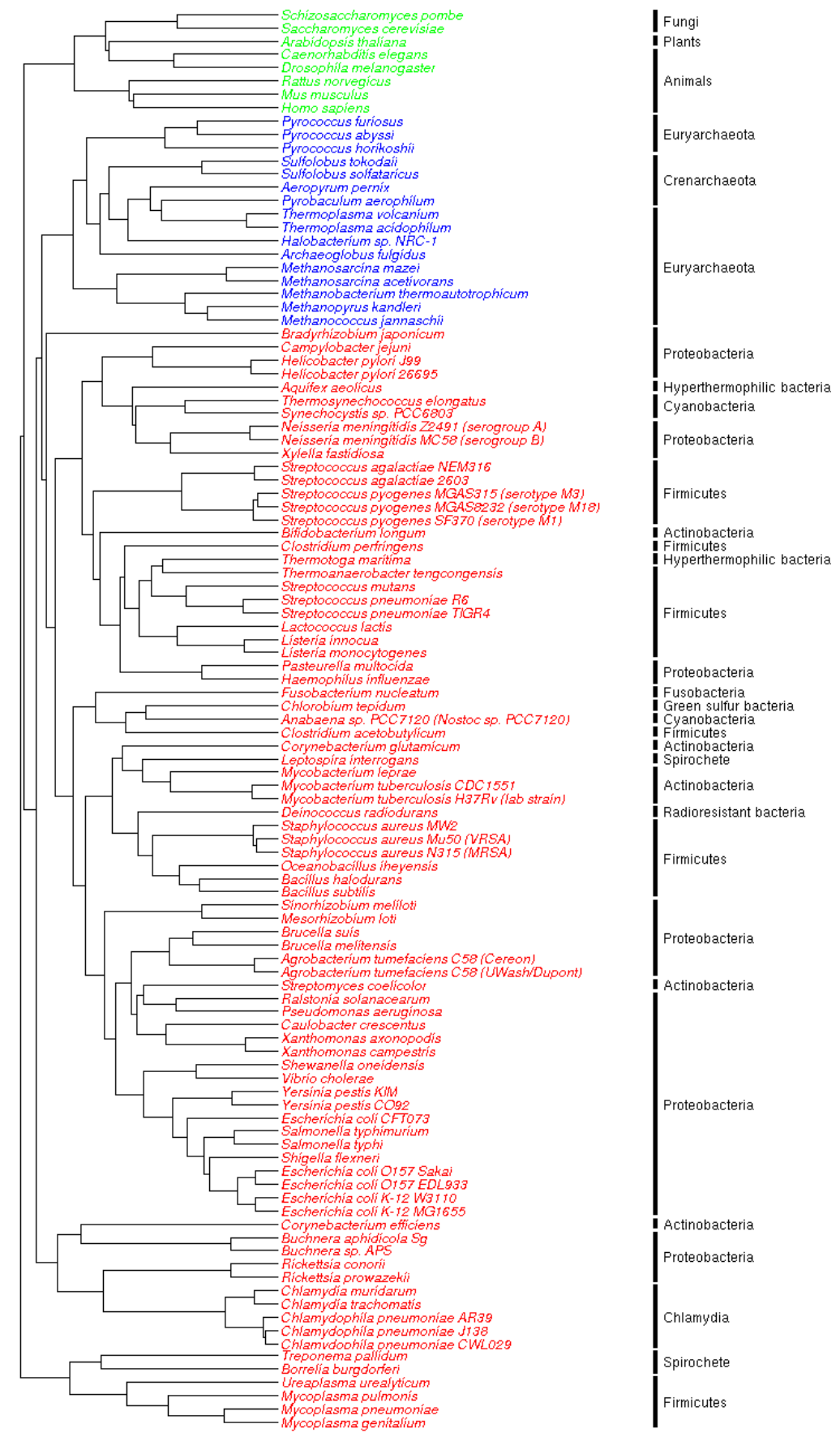

FIG. 2: A phylogenetic tree generated using NCD applied to all pairs of metabolic network structures from 107 organisms in KEGG. Different domains of life appear in distinct branches. Bacteria are shown in red, archaea in blue, and eukaryotes in green. Subclasses of species within each domain are labeled on the right. Parasitic bacteria (bottom branch) are separated from the rest as observed earlier. This separation of the domains of life indicates that the method is able to discover the fundamental structural differences in the metabolic networks. 


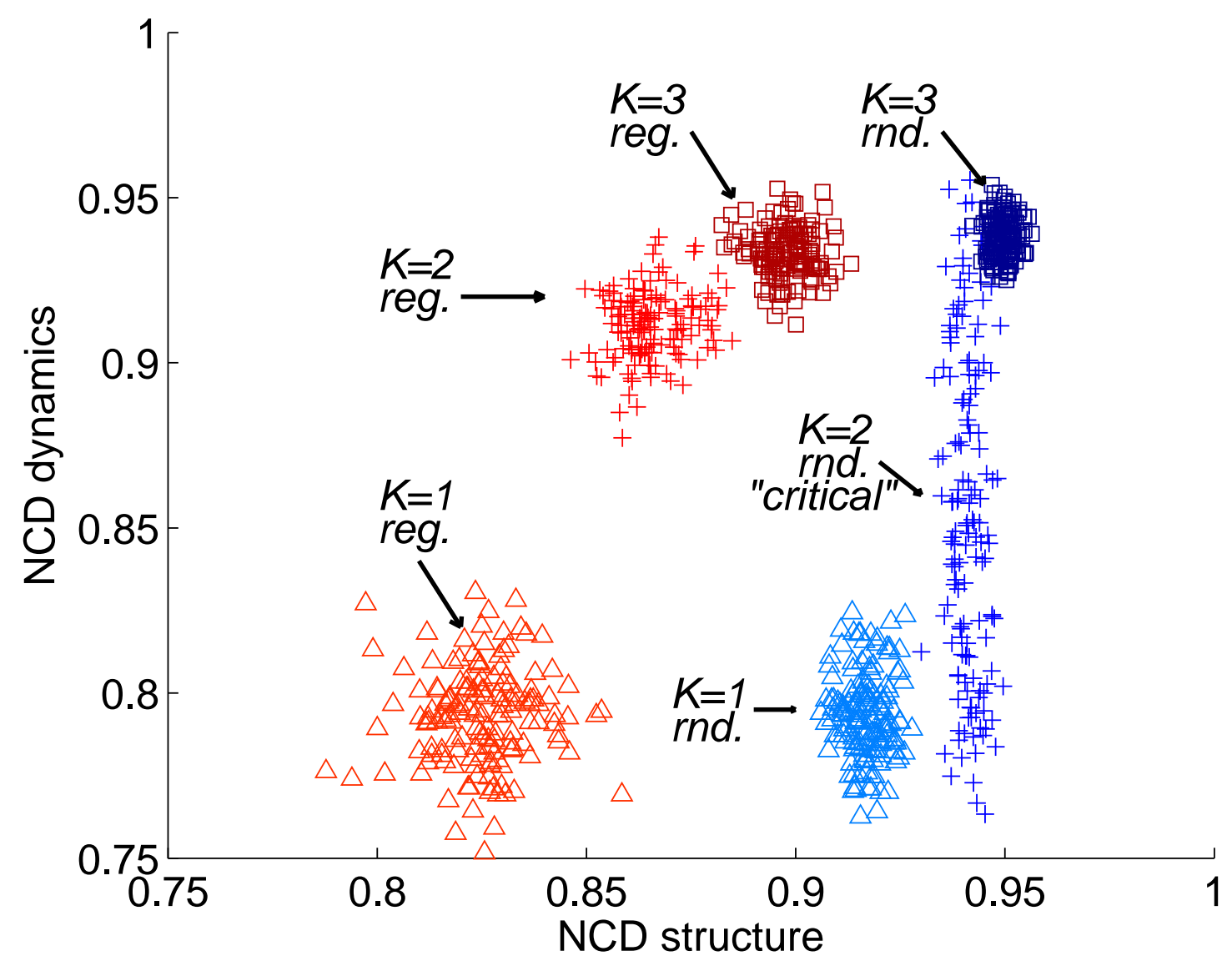

FIG. 3: The normalized compression distance (NCD) applied to network structure and dynamics. Six ensembles of random Boolean networks $(K=1,2,3$ each with random or regular topology; $N=1000)$ were used to generate 150 networks from each ensemble. NCDs were computed between pairs of networks (both chosen from the same ensemble) based on their structure (x-axis) and their dynamic state trajectories (y-axis). Different ensembles are clearly distinguishable. The critical ensemble is more elongated, implying diverse dynamical behavior. 
[1] Y. D. Nochomovitz and H. Li, Proc. Natl. Acad. Sci. USA 103, 4180 (2006).

[2] E. A. Variano, J. H. McCoy, and H. Lipson, Phys. Rev. Lett. 92 (2004).

[3] M. Aldana and P. Cluzel, Proc. Natl. Acad. Sci. USA 100, 8710 (2003).

[4] R. Albert, H. Jeong, and A.-L. Barabási, Nature 406, 378 (2000).

[5] S. A. Kauffman, C. Peterson, B. Samuelsson, and C. Troein, Proc. Natl. Acad. Sci. USA 100, 14796 (2003).

[6] I. Shmulevich, H. Lähdesmäki, E. R. Dougherty, J. Astola, and W. Zhang, Proc. Natl. Acad. Sci. USA 100, 10734 (2003).

[7] S. A. Kauffman, Physica A 340, 733 (2004).

[8] A.-L. Barabási and R. Albert, Science 286, 509 (1999).

[9] D. J. Watts and S. H. Strogatz, Nature 393, 440 (1998).

[10] S. A. Kauffman, The Origins of Order: Self-organization and selection in evolution (Oxford University Press, New York, 1993).

[11] K. Klemm and S. Bornholdt, Proc. Natl. Acad. Sci. USA 102, 18414 (2005).

[12] M. Aldana, S. Coppersmith, and L. P. Kadanoff, in Perspectives and Problems in Nonlinear Science, edited by E. Kaplan, J. E. Marsden, and K. R. Sreenivasan (Springer-Verlag, New York, 2003), pp. 23-89.

[13] B. Derrida and Y. Pommeau, Europhys. Lett. 1, 45 (1986).

[14] T. Mestl, R. J. Bagley, and L. Glass, Phys. Rev. Lett. 79, 653 (1997).

[15] I. Shmulevich, S. A. Kauffman, and M. Aldana, Proc. Natl. Acad. Sci. USA 102, 13439 (2005).

[16] P. Rämö, J. Kesseli, and O. Yli-Harja, J. Theor. Biol. 242, 164 (2006).

[17] P. Krawitz and I. Shmulevich, Phys. Rev. Lett. 98 (2007).

[18] P. Rämö, S. A. Kauffman, J. Kesseli, and O. Yli-Harja, Physica D 227, 100 (2007).

[19] B. Luque and A. Ferrera, Complex Systems 12, 241 (2000).

[20] R. V. Solé, S. Manrubia, B. Luque, J. Delgado, and J. Bascompte, Complexity 1, 13 (1996).

[21] C. G. Langton, Physica D 42, 12 (1990).

[22] N. H. Packard, in Dynamic Patterns in Complex Systems, edited by A. J. Mandell, J. A. S. Kelso, and M. F. Shlesinger (World Scientific, Singapore, 1988), pp. 293 - 301.

[23] M. Li and P. Vitanyi, An Introduction to Kolmogorov Complexity and Its Applications 
(Springer-Verlag, New York, 1997), 2nd ed.

[24] C. H. Bennett, P. Gacs, M. Li, P. M. B. Vitanyi, and W. Zurek, IEEE Trans. Inform. Theory 44, 1407 (1998).

[25] M. Li, X. Chen, X. Li, B. Ma, and P. Vitanyi, IEEE Trans. Inform. Theory 50, 3250 (2004).

[26] R. Cilibrasi and P. Vitanyi, IEEE Trans. Inform. Theory 51, 1523 (2005).

[27] S. Kauffman, Nature 224, 177 (1969).

[28] M. Kanehisa and S. Goto, Nucleic Acids Res. 28, 27 (2000).

[29] J. Podani, Z. N. Oltvai, H. Jeong, B. Tombor, A.-L. Barabási, and E. Szathmáry, Nat. Genet. 29, 54 (2001). 